\title{
Victimización infantil, un problema de salud en el sistema social capitalista
}

\author{
Child victimization, a health problem in the capitalist social \\ system
}

\author{
Florángel Urrusuno Carvajal'; Roberto Rodríguez Fernández" \\ 'Especialista de II Grado en Medicina General Integral. Máster en Atención Primaria \\ de Salud. Facultad de Ciencias Médicas "Manuel Fajardo". La Habana, Cuba. \\ "Licenciado en Ciencias Sociales. Dirección Nacional de Tránsito. La Habana, Cuba.
}

\section{RESUMEN}

La victimización infantil es un fenómeno complejo de la sociedad conocido desde hace siglos. Hacia 1946, se crea la UNICEF, como primer organismo internacional para proteger la infancia, siendo esta la base para llegar a la Convención sobre los Derechos del Niño y posteriormente a la Cumbre sobre la Infancia de 1990. El incremento de la victimización infantil está estrechamente vinculado a la globalización que vive el mundo. La violencia infantil está asociada a la falta de preocupación de los estados por legislar primeramente y luego hacer cumplir las leyes que protejan a los infantes. Se tipifican algunos delitos contra los menores así como las diferentes modalidades de explotación infantil: tráfico de menores, la pornografía y prostitución infantil así como el contrabando de órganos. Se plantea que en la inmensa mayoría de los países del mundo, el trabajo no constituye una forma de capacitación y sin duda, una forma de explotación y violación de los derechos del niño. La violencia se manifiesta independientemente del sexo, raza o precedencia étnica, produciéndose de manera indistinta el abuso sexual, el físico o el emocional. La violencia contra la infancia está presente en todas las esferas de la sociedad capitalista haciéndose más crítica en los países del tercer mundo. Las acciones emprendidas por Cuba, Venezuela y otros, son ejemplos de cuanto se alcanza con mucha voluntad política y pocos recursos en la defensa de los derechos del niño.

Palabras clave: Victimización infantil, sistema social capitalista. 


\begin{abstract}
The child victimisation is a complex phenomenon of the society known for centuries. In 1946, UNICEF was created as first international organization to protect childhood, being the basis for the Convention on the Rights of Childhood and subsequently the Summit on the Childhood in 1990. The increased child victimisation is closely linked to the globalization. The child violence is associated to lack of concern by the states regarding legislation and putting into effect laws that protect the infants. This article categorizes some crimes against children as well as different forms of child exploitation (children traffic, infant pornography and child prostitution as well as organ smuggling.) It is stated that in most of the countries, child work is not a way of training but exploitation and violation of the child's rights. Violence is present regardless of sex, race or ethnic origin leading to sexual abuse. Violence against children is present in all the spheres of the capitalist society, being more acute in the Third World countries. The actions undertaken by Cuba, Venezuela and others are examples of the achievements that can be attained in the defense of the children's rights with much political willingness and few resources.
\end{abstract}

Key words: Child victimization, capitalist social system.

\title{
I NTRODUCCI ÓN
}

Los crímenes contra menores existieron siempre. El niño igual que la mujer, antaño fueron considerados meros objetos, no personas con plenos derechos.

A través de la historia, merced a los cambios sociales y culturales el niño comienza a ser visto paulatinamente como individuo. Luego de la Segunda Guerra Mundial, hacia 1948, se crea la UNICEF, como primer organismo internacional que centra su atención en la infancia, siendo esta la base para llegar al día 20 de Noviembre de 1989 a la Convención sobre los Derechos del Niño y posteriormente el 2 de Septiembre de 1990 en New York se efectúa la Cumbre Mundial a favor de la Infancia, de resultados aceptados por casi todos los estados miembros exceptuando Los Estados Unidos (EE.UU.), Somalia y otros.

\section{CTI MI ZACI ÓN I NFANTI L}

La victimización infantil es un fenómeno complejo de la sociedad, conocido desde hace siglos, sin distinción de grupos de edad, color de la piel, sexo y situaciones económicas: generalmente los pobres son los más afectados. Se plantea que es más frecuente que muchas enfermedades y menos diagnosticada, afecta a un porcentaje elevadísimo de la población y conduce a altos costos en la sociedad.

Dada la situación crítica de la infancia, en el mundo hay millones de niños que son víctimas y sufren la ausencia de leyes que protejan su desarrollo, su integridad física y moral. Esta victimización está asociada a una globalización unipolar neoliberal y violencia sin precedente en la historia de la humanidad. 
Los problemas que genera la globalización económica neoliberal y la pobreza en la familia, a partir de la negligencia de los estados y sus gobiernos cuando violan los derechos de los ciudadanos establecidos en sus propias leyes, incumplen los acuerdos internacionales, se suman a la política exigente de una "minoría élite" que controla la estructura de los estados en contra del bienestar de su propio país, de aquí que la formación de valores como son la salud, la educación y la seguridad social no son elementos prioritarios dentro de los programas de estas naciones. Sus estructuras socioeconómicas comprometidas con las grandes potencias permiten las enormes injusticias sociales e iniquidad entre los diferentes grupos de poblaciones; además estimula la corrupción, las prebendas y el tráfico de narcóticos y personas. Se carece de ética y voluntad política, elementos fundamentales en la formación de valores y en la defensa de los derechos del niño.

Por ello no resulta extraño que en la inmensa mayoría de los países del mundo, los niños son víctimas de la pobreza que genera las políticas neoliberales, con las insuficiencias educacionales y de salud, que los obliga a trabajar desde muy pequeño, niños que son abandonados por su familia y son víctimas de la explotación sexual, las drogas, la violencia callejera y de estado.

Los tipos de delitos que se cometen contra los menores son muy variados y la responsabilidad del adulto en ellos se puede tipificar según su gravedad. Para ello se parte de aquellos delitos que atentan contra la integridad psicofísica y la vida del niño y que se expresan desde la gestación hasta el maltrato en el seno familiar, con secuelas de lesiones que pueden llegar en oportunidades a la muerte.

La creciente complejidad de la vida moderna muestra modalidades de explotación infantil hasta hace poco, escasamente conocidas, entre ellas, el tráfico de niños, pornografía infantil, turismo sexual y la prostitución infantil.

El tráfico de niños es el traslado de menores de un país a otro (internacional) o dentro de un mismo país (nacional) con fines ilícitos; niños que son separados de sus familias con fines de lucro, ya sea para beneficio de individuos, de grupos o de organizaciones.

Esto sucede tanto en los países desarrollados como en los subdesarrollados; la causa principal en estos últimos es la pobreza. El trabajo no beneficia económicamente al menor ni constituye una forma de capacitación sino que reviste formas de explotación y violación a sus derechos, ya que en esos casos son confinados, por ejemplo, a talleres o burdeles. ${ }^{1}$

En el contexto actual los efectos negativos de la crisis económica y financiera en EE.UU. se acrecientan a escala global, se ha derrumbado el mercado financiero norteamericano y esa realidad fluye hacia el exterior, lo que da lugar a la mayor crisis capitalista desde la ocurrida en 1929.

La actual situación ha derribado los mitos de la desregulación del mercado, de la llamada economía de casino y del crédito ilimitado, traducidos en deudas impagables de instituciones, del gobierno y de las personas. Esto fue alertado por el Comandante en J efe Fidel Castro Ruz en diferentes foros internacionales, y en sus reflexiones cuando vaticinó que la crisis económica sería mucho mas profunda, alertó de los problemas que se producirían al no cuidar los ecosistemas, llamó la atención del uso de los alimentos como combustibles para paliar la aguda crisis energética y que esto traería también una crisis alimentaría; como es lógico las clases sociales de menos recursos y los países más pobres son precisamente los mas afectados y dentro de estos los niños que son los más vulnerables. ${ }^{2}$ 
La voluntad política de estos gobiernos no está encaminada a desarrollar redes de vigilancia ni planes de intervención y no tienen en cuenta a los niños, antes, durante y después de que se produzca cualquier situación negativa para con ellos.

Las estadísticas reportadas por las agencias de servicios de protección de menores a lo largo de los EE.UU. reciben más de 50000 denuncias semanales de sospechas de violencias a niños, tres millones de ellos esperan ser elegidos por el medicaid.

En el 2001, se recibieron casi tres millones de denuncias de sospecha de abuso, contra aproximadamente cinco millones de niños. En aproximadamente dos tercios ( $67 \%$ ) de estos casos, la información proporcionada en la denuncia fue suficiente para dar lugar a una investigación.

Como resultado de estas investigaciones, se encontró que aproximadamente 903 000 niños fueron víctimas del abuso o negligencia.

Más de la mitad ( $59 \%$ ) de las víctimas sufrieron por abandono, esto significa que una persona encargada del niño o niña no satisfizo las necesidades básicas del menor. En comparación, el número de víctimas de abuso físico fue menor ( $19 \%$ ) y aún menor fue el número de víctimas de abuso sexual ( $10 \%$ ), aunque estos casos tienen ciertamente más cobertura y publicidad. El menor número de victimas ( 7 \%) correspondió al abuso emocional, el cual incluye rehusarse a alentar a un niño, criticarlo, o rechazarlo. Trágicamente, un promedio de tres niños muere cada día como resultado del abuso y la negligencia. ${ }^{1}$

Ningún grupo de niños es inmune. Sin diferencias, tanto los niños como las niñas pueden sufrir negligencia y abuso físico. Niños de todas las razas y procedencias étnicas son víctimas del maltrato. En 2001, la mitad de todas las víctimas reportadas eran de raza blanca ( $50 \%$ ), la cuarta parte ( $25 \%$ ), africanosamericanos y el $15 \%$, de procedencia hispana. Los indios americanos (nativos de Alaska) representaban el $2 \%$ de las víctimas, y los isleños asiáticos y del Pacífico, el $1 \%$.

Los niños de todas edades sufren abuso, pero los niños menores son los más vulnerables. Los menores de un año representaron el $41 \%$ de las muertes reportadas en el 2001 , el $8 \%$ de los niños que murieron eran menores de seis años de edad; 130 millones de niños en el mundo son analfabetos.

Mientras que en los países desarrollados la expectativa de vida es de 80 años, en los países pobres sólo llegan a 40; se estiman que 1 millón muere antes de cumplir la semana de nacidos, otros 4 millones mueren antes de cumplir el mes y además se calcula que 10 millón de niños mueren antes de cumplir los 5 años de vida. Entre tanto otros piensan en como enriquecerse a costa de la guerra. ${ }^{3}$

En América Latina se estima que 83 millones de personas viven en la pobreza, existe 50 millones de niños de la calle, entre el 5 y el $15 \%$ de la población callejera está constituido por niños que han abandonado totalmente el hogar y se han incorporado a la subcultura de la calle en donde la violencia, los malos tratos, la delincuencia y el uso de las drogas, forman parte de la vida cotidiana.

Las perspectivas de los primeros años del nuevo milenio nada bueno ofrece, no obstante los pueblos empiezan a darse cuenta que la única solución para ellos y muchos otros es la que Cuba y Venezuela llevan adelante rescatando la dignidad del ser humano. En América Latina y el Caribe ahora son más los países que se están sumando y empiezan a reconocer el valor de la infancia como algo que hay 
que salvar si se quiere salvar a la humanidad. Ellos son las futuras generaciones que tendrán la importante tarea de llevar adelante el desarrollo sostenible y preservar la especie. En este noble empeño se está demostrando que la voluntad política es el elemento fundamental para alcanzar el sueño de los países más pobres del planeta de tener una vida más plena, justa y digna a la cual se unen otros factores como la integración de los países con iguales características, en intercambio mutuo de recursos humanos y materiales sin que medien los intereses privados. Esta unidad permitirá abordar de modo más eficiente las tareas que propicien la disminución paulatina de la violencia infantil, entre ellas, darle solución a un grupo de problemas de carácter económico y social, el aprendizaje del comportamiento particular, el fortalecimiento del papel que desempeñan los medios de difusión, el personal de la salud, la educación y el resto de la sociedad; en la conducción adecuada de este fenómeno en la comunidad como poleas trasmisoras de la política del estado en defensa de los intereses del niño.

\section{REFERENCI AS BI BLI OGRÁFI CAS}

1. Saniuk J M. El rol de la División Unidad Especializada de Investigación de Crímenes contra Menores [sitio en Internet]. [actualizado 2000, citado 20 Ene 2008]; Año1(1). Disponible en: http://www.salvador.edu.ar/vrid/di/r_pub00.htm

2. Castro Ruz F. Ike Financiero. Granma. Sept 2008: 1.

3. Acosta TN. Maltrato Infantil. Prevención. 3ra ed. [sitio en Internet]. [actualizado 2008, citado 20 Ene 2008]. Disponible en:

http://www.prevemi.sld.cu/diplomado/V_diplomado/discusion_negligencia.html

Recibido: 30 de octubre de 2008.

Aprobado: 22 de noviembre de 2008.

Florángel Urrusuno Carvajal. Facultad de Ciencias Médicas "Manuel Fajardo". La Habana, Cuba.

E-mail: florangel.urrusuno@infomed.sld.cu 